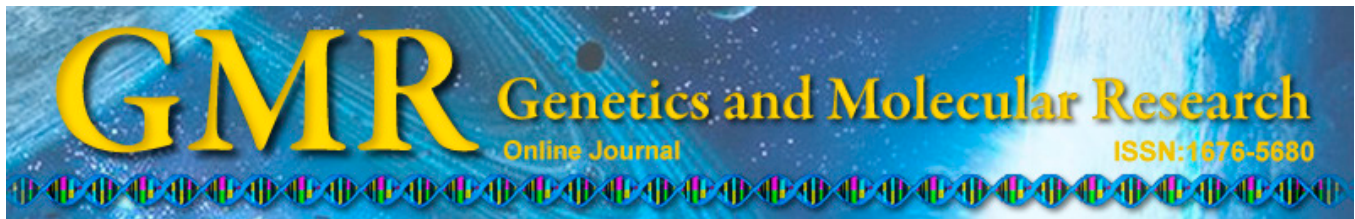

\title{
Genetic analysis of maize kernel thickness by quantitative trait locus identification
}

\author{
S.S. Wen', G.Q. Wen², C.M. Liao ${ }^{3}$ and X.H. Liu' \\ ${ }^{1}$ Key Laboratory of Southwest Rice Biology and Genetic Breeding, \\ Institute of Rice and Sorghum, Sichuan Academy of Agricultural Sciences, \\ Ministry of Agriculture, Luzhou Branch of National Rice Improvement Center, \\ Luzhou, China \\ ${ }^{2}$ Key Laboratory of Southwest China Wildlife Resources Conservation, \\ Ministry of Education, College of Life Science, \\ China West Normal University, Nanchong, China \\ ${ }^{3}$ Library, China West Normal University, Nanchong, China \\ Corresponding author: X.H. Liu \\ E-mail:350783409@qq.com
}

Genet. Mol. Res. 14 (3): 9858-9864 (2015)

Received December 9, 2014

Accepted May 8, 2015

Published August 19, 2015

DOI http://dx.doi.org/10.4238/2015.August.19.19

\begin{abstract}
Kernel thickness is one of the most important traits in kernel structure, and is related to yield. To ascertain its genetic information more clearly, an immortal recombinant inbred line segregation population was used to map the quantitative trait loci (QTLs) for kernel thickness. As a result, two QTLs were identified on chromosome 9; both of them had negative additive effects, and could decrease kernel thickness to some extent. The QTLs explained 25.8\% of the total phenotypic variation. These results advance our understanding of the genetic basis of kernel thickness in maize-breeding programs.
\end{abstract}

Key words: Maize (Zea mays L.); Quantitative trait locus; Recombinant inbred line; Kernel thickness 


\section{INTRODUCTION}

Maize (Zea mays L.) is one of the most important global cereal crops, is widely consumed, and plays a crucial role in maintaining food security. In addition, maize is used as a raw material in industry. Its high demand makes grain yield a major target of maize breeding (Liu et al., 2014). Maize grain yield is a complex, quantitative genetic trait, and can be divided into smaller components, such as plant number per hectare, kernel number per ear, 100-kernel weight, etc. Kernel thickness is an important agronomic trait, which is related to 100 -kernel weight and kernel appearance.

Quantitative trait locus (QTL) mapping is an effective means of understanding the genetic mechanisms of agronomic traits. Numerous studies on QTL identification in crops have been conducted, including in maize (Riedelsheimer et al., 2012; Almeida et al., 2014; Herzog et al., 2014), rice (Chen et al., 2014; Xu et al., 2014; Zhou et al., 2014), wheat (Bennett et al., 2012; Ishikawa et al., 2014; Katkout et al., 2014), sorghum (Rajkumar et al., 2013; Alam et al., 2014), and soybean (Liang et al., 2014; Mamidi et al., 2014). Regarding maize kernel-related traits, most studies that have used QTL mapping have focused on kernel number per ear (Liu et al., 2011; Zhang et al., 2013; Li et al., 2014) or kernel weight (Tang et al., 2010; Ding et al., 2011; Prado et al., 2014). There have only been a few studies conducted on kernel thickness. Li et al. (2009) identified four QTLs on chromosomes 2, 3, 6, and 10, with one on every chromosome, and Peng et al. (2011) mapped three QTLs (using a population derived from Qi319 and Huangzaosi) on chromosomes 2, 3, and 10. Peng et al. (2011) used another segregation population bred from Ye478 and Huangzaosi, and mapped three QTLs on chromosomes 1, 3, and 10. Recently, Zhang et al. (2014) and Liu et al. (2014) found 11 and 18 QTLs, respectively, for kernel thickness. These different results were probably caused by the use of different parental materials, segregation populations, genetic maps, or ecological environments. Therefore, it is significant to select a new population to study the genetic mechanism of kernel thickness by QTL mapping in maize-breeding program.

In the present study, an immortal recombinant inbred line (RIL) population derived from the parental inbred lines Mo17 and Huangzaosi was used in QTL detection for kernel thickness; its aims were to understand the genetic basis of kernel thickness more clearly, and to locate the quantitative loci that are associated with kernel thickness in maize.

\section{MATERIAL AND METHODS}

\section{Experimental materials}

We used the two parental inbred lines Mo17 and Huangzaosi and an RIL segregation population consisting of 239 RILs derived from the two parents. Mo17 and Huangzaosi are representative inbred lines of the Lancaster (United States) and Tangsipingtou (China) heterosis groups, respectively.

\section{Field design and phenotypic observations}

The 241 maize materials were sown at the Nanchong Agricultural Academy, Nanchong City, Sichuan Province, China. Twenty plants of each of three replicates were designed 
for every maize line. After harvesting, the thickness of 20 randomly selected kernels from each line was measured with an electronic digital caliper, and Microsoft Excel 2010 was used to compute the mean thickness of the 20 maize kernels.

\section{Descriptive statistics}

The mean kernel thicknesses of the parents were compared, and descriptive statistics of the RILs were obtained using SPSS version 11.5, including ranges, minima, maxima, means, standard deviations (SD), skewness, kurtosis, and coefficients of variation (COV); frequency distributions of kernel thickness were also generated.

\section{QTL detection}

An established marker linkage map (Liu et al., 2009) that included 100 simple-sequence repeat (SSR) markers and covered $1421.5 \mathrm{cM}$ was used for QTL mapping for kennel thickness, based upon the descriptive statistics of the RILs. The QTLs for kernel thickness were detected by the interval mapping method, using the QTL mapping software Windows QTL Cartographer version 2.5 (Wang et al., 2010), with $1.0 \mathrm{cM}$ of walk speed. The $\log 10$ of the odds ratio (LOD) threshold value used to detect QTLs was determined by a 1000-permutation test $(\alpha=0.05)$ (Doerge and Churchill, 1996). The chromosomal positions, additive genetic effects, and percentages of phenotypic variance of the QTLs that were identified were estimated using the same QTL mapping software. The QTLs were then mapped onto the marker linkage map using the MapChart 2.1 software (Voorrips, 2002).

\section{RESULTS}

\section{Descriptive statistics of parents and RILs}

On average, Huangzaosi had thicker kernels than Mo17 (Table 1). Kernel thickness in the RILs ranged between 4.08 and $8.53 \mathrm{~mm}$ (Table 2) and followed a normal distribution (Figure 1), indicating that it is a quantitative trait that is controlled by several genes.

\section{Table 1. Kernel thickness in maize parental inbred lines.}

\begin{tabular}{lc}
\hline Parental inbred line & Kernel thickness $(\mathrm{mm})$ \\
\hline Mo17 & 4.77 \\
Huangzaosi & 5.43 \\
\hline
\end{tabular}

Table 2. Descriptive statistics for kernel thickness in maize recombinant inbred lines.

\begin{tabular}{lcccccccc}
\hline Trait & Range $(\mathrm{mm})$ & Minimum $(\mathrm{mm})$ & Maximum $(\mathrm{mm})$ & Mean $(\mathrm{mm})$ & SD & Skewness & Kurtosis & COV $(\%)$ \\
\hline Kernel thickness & 4.45 & 4.08 & 8.53 & 5.28 & 0.59 & 0.875 & 3.309 & 11.17 \\
\hline
\end{tabular}

$\mathrm{SD}$, standard deviation; $\mathrm{CV}$, coefficient of variation. 


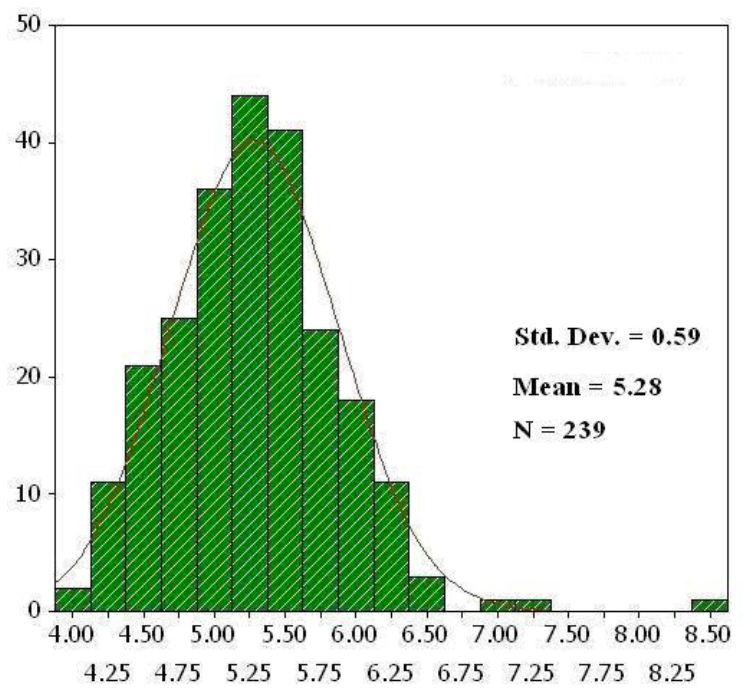

Figure 1. Frequency distribution of kernel thickness in maize recombinant inbred lines.

\section{QTL detection}

The results of the QTL scanning are shown in Figure 2. According to the LOD threshold value of 2.20, only two QTLs ( $q K T 9-1$ and $q K T 9-2)$ were detected, which were on chromosome 9 (Figure 3). The two QTLs had the same adjacent marker (Umc1357), but $q$ KT9-1 was closer to it $(2.1 \mathrm{cM})$ than $q K T 9-2(10.0 \mathrm{cM}) . q K T 9-1$ was between Phi016 and Umc1357, and $q$ KT9-2 was between Umc1357 and Bnlg1375 (Table 3). Both QTLs exhibited negative additive effects, suggesting that they decrease kernel thickness to some extent. qKT9-1 accounted for $10.5 \%$ of the total phenotypic variation, and 9KT9-2 accounted for up to $15.3 \%$. Therefore, the two QTLs identified in this study are different chromosomal loci, despite having the same adjacent marker and being in close proximity to each other.

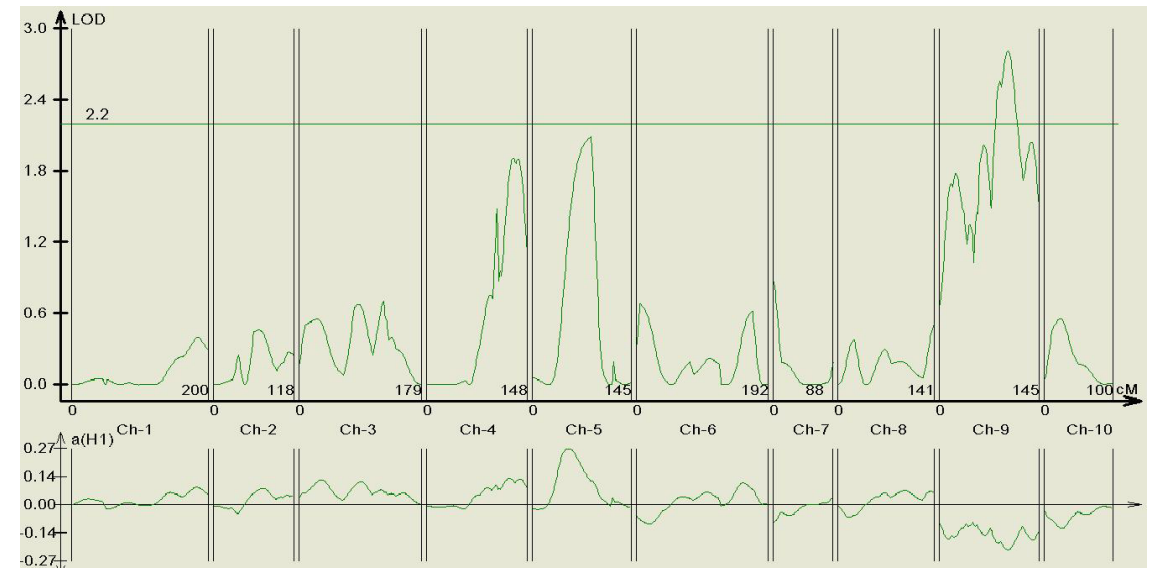

Figure 2. Quantitative trait locus (QTL) analysis result by interval mapping for maize kernel thickness. Only two QTLs were detected on chromosome 9 according to the $\log 10$ of the odds ratio (LOD) curves. 


\section{Chromosome 9}

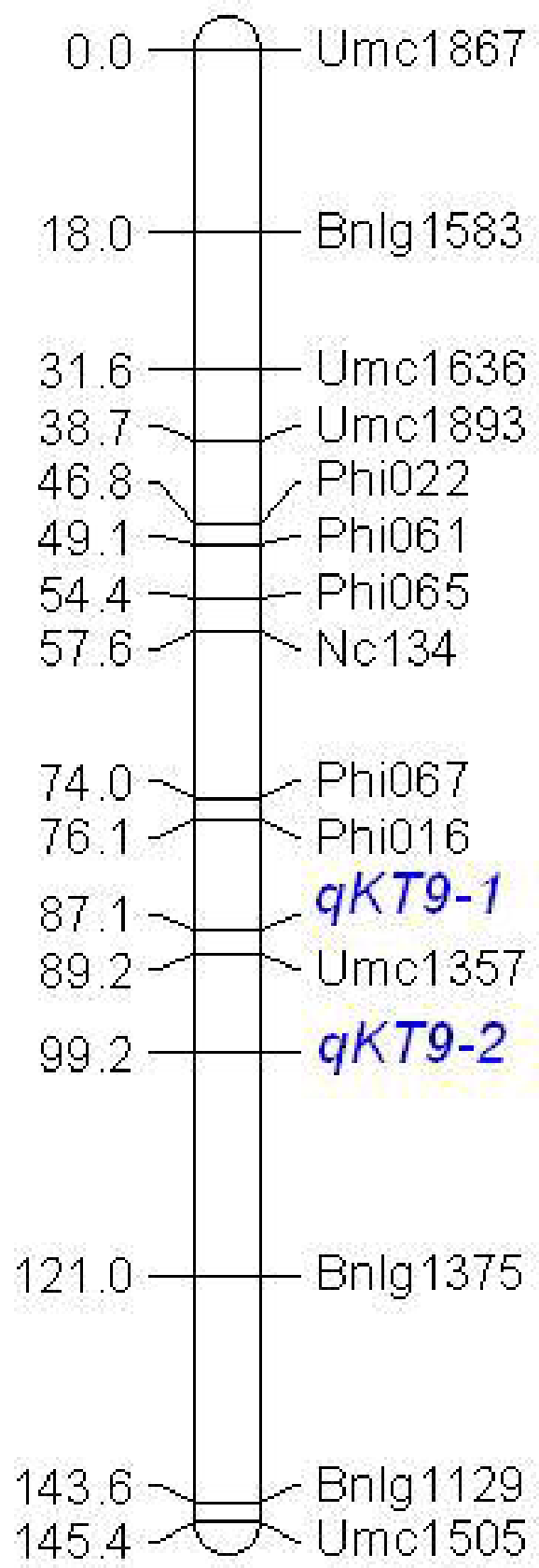

Figure 3. Chromosomal distribution of quantitative trait loci (QTLs). Two QTLs were mapped on chromosome 9, one between Phi016 and Umc1357 (qKT9-1) and the other between Umc1357 and Bnlg1375 (qKT9-2). 
Table 3. Quantitative trait loci associated with maize kernel thickness and their genetic parameters.

\begin{tabular}{lcccccc}
\hline QTL & Chr. & Chromosomal position $(\mathrm{cM})$ & Genetic distance to Umc1357 (cM) & LOD & Additive effect & $R^{2}(\%)$ \\
\hline qKT9-1 & 9 & 87.1 & 2.1 & 2.6 & -0.18 & 10.5 \\
qKT9-2 & 9 & 99.2 & 10.0 & 2.8 & -0.22 & 15.3 \\
Total & & & & & 25.8 \\
\hline
\end{tabular}

QTL, quantitative trait locus; Chr., chromosome number; LOD, $\log 10$ of the odds ratio; $R^{2}$, percentage of phenotypic variance explained by QTL.

\section{DISCUSSION}

We found two QTLs, both of them on chromosome 9. They both exhibited negative additive effects, and could decrease kernel thickness to some extent.

Previous studies have found several QTLs for kernel thickness, with at least one on each of the 10 maize chromosomes (Table 4). Liu et al. (2014) identified up to 18 QTLs on seven chromosomes, and Zhang et al. (2014) found 11 on six chromosomes. These results suggest that kernel thickness is a quantitative trait and is controlled by several genes. Our results confirm the findings of Liu et al. (2014), who also found two QTLs on chromosome 9. However, the two results differ: the two QTLs identified by Liu et al. (2014) exhibited positive additive effects, whereas the two mapped in our study exhibited negative additive effects.

Table 4. Quantitative trait loci associated with maize kernel thickness and their chromosomal distribution.

\begin{tabular}{|c|c|c|c|c|c|c|c|c|c|c|c|c|}
\hline \multirow[t]{2}{*}{ Reference } & \multirow[t]{2}{*}{ Parental material } & \multirow[t]{2}{*}{ No. of mapped QTLs } & \multicolumn{10}{|c|}{ Chr. No. } \\
\hline & & & 1 & 2 & 3 & 4 & 5 & 6 & 7 & 8 & 9 & 10 \\
\hline Li et al. (2009) & Qi319 and Huangzaosi & 4 & & 1 & 1 & & & 1 & & & & 1 \\
\hline Peng et al. (2011) & Qi319 and Huangzaosi & 3 & & 1 & 1 & & & & & & & 1 \\
\hline Peng et al. (2011) & Ye478 and Huangzaosi & 3 & 1 & & 1 & & & & & & & 1 \\
\hline Zhang et al. (2014) & Xu178 and HuangC & 11 & 1 & 3 & 1 & & & 2 & 1 & 3 & & \\
\hline Liu et al. (2014) & V671 and Mc & 18 & 5 & 1 & & 2 & 3 & & & 2 & 2 & 3 \\
\hline This study & Mo17 and Huangzaosi & 2 & & & & & & & & & 2 & \\
\hline
\end{tabular}

QTL, quantitative trait locus; Chr. No., chromosome number.

Although we detected two QTLs in maize that were associated with kernel thickness, they were relatively far from their adjacent SSR marker (Umc1357) in genetic distance, so more molecular markers need to be added to this chromosomal region in order to more finely map them, and this study is currently ongoing.

\section{Conflicts of interest}

The authors declare no conflict of interest.

\section{ACKNOWLEDGMENTS}

Research supported by the Scientific Research Fund of Sichuan Provincial Education Department (\#13ZA0012).

\section{REFERENCES}

Alam MM, Mace ES, van Oosterom EJ, Cruickshank A, et al. (2014). QTL analysis in multiple sorghum populations 
facilitates the dissection of the genetic and physiological control of tillering. Theor. Appl. Genet. 127: 2253-2266.

Almeida GD, Nair S, Borém A, Cairns J, et al. (2014). Molecular mapping across three populations reveals a QTL hotspot region on chromosome 3 for secondary traits associated with drought tolerance in tropical maize. Mol. Breed. 34 : 701-715.

Bennett D, Reynolds M, Mullan D, Izanloo A, et al. (2012). Detection of two major grain yield QTL in bread wheat (Triticum aestivum L.) under heat, drought and high yield potential environments. Theor. Appl. Genet. 125: 14731485.

Chen JY, Guo L, Ma H, Chen YY, et al. (2014). Fine mapping of $q H d 1$, a minor heading date QTL with pleiotropism for yield traits in rice (Oryza sativa L.). Theor. Appl. Genet. 127: 2515-2524.

Ding JQ, Ma JL, Zhang CR, Dong HF, et al. (2011). QTL mapping for test weight by using $\mathrm{F}_{2: 3}$ population in maize. $J$. Genet. 90: 75-80.

Doerge RW and Churchill GA (1996). Permutation tests for multiple loci affecting a quantitative character. Genetics 142: 285-294.

Herzog E, Falke KC, Presterl T, Scheuermann D, et al. (2014). Selection strategies for the development of maize introgression populations. PLoS One 9: e92429.

Ishikawa G, Nakamura K, Ito H, Saito M, et al. (2014). Association mapping and validation of QTLs for flour yield in the soft winter wheat variety Kitahonami. PLoS One 9: e111337.

Katkout M, Kishii M, Kawaura K, Mishina K, et al. (2014). QTL analysis of genetic loci affecting domestication-related spike characters in common wheat. Genes Genet. Syst. 89: 121-131.

Li F, Jia HT, Liu L, Zhang CX, et al. (2014). Quantitative trait loci mapping for kernel row number using chromosome segment substitution lines in maize. Genet. Mol. Res. 13: 1707-1716.

Li YX, Wang Y, Shi YS, Song YC, et al. (2009). Correlation analysis and QTL mapping for traits of kernel structure and yield components in maize. Sci. Agric. Sin. 42: 408-418.

Liang H, Yu Y, Yang H, Xu L, et al. (2014). Inheritance and QTL mapping of related root traits in soybean at the seedling stage. Theor. Appl. Genet. 127: 2127-2137.

Liu XH, Tan ZB and Rong TZ (2009). Molecular mapping of a major QTL conferring resistance to SCMV based on immortal RIL population in maize. Euphytica 167: 229-235.

Liu XH, He SL, Zheng ZP, Tan ZB, et al. (2011). Genetic loci mapping associated with maize kernel number per ear based on a recombinant inbred line population grown under different nitrogen regimes. Genet. Mol. Res. 10: 3267-3274.

Liu Y, Wang L, Sun C, Zhang Z, et al. (2014). Genetic analysis and major QTL detection for maize kernel size and weight in multi-environments. Theor. Appl. Genet. 127: 1019-1037.

Mamidi S, Lee RK, Goos JR and McClean PE (2014). Genome-wide association studies identifies seven major regions responsible for iron deficiency chlorosis in soybean (Glycine max). PLoS One 9: e107469.

Peng B, Li YX, Wang Y, Liu C, et al. (2011). QTL analysis for yield components and kernel-related traits in maize across multi-environments. Theor. Appl. Genet. 122: 1305-1320.

Prado SA, López CG, Senior ML and Borrás L (2014). The genetic architecture of maize (Zea mays L.) kernel weight determination. G3 4: 1611-1621.

Rajkumar, Fakrudin B, Kavil SP, Girma Y, et al. (2013). Molecular mapping of genomic regions harbouring QTLs for root and yield traits in sorghum (Sorghum bicolor L. Moench). Physiol. Mol. Biol. Plants 19: 409-419.

Riedelsheimer C, Technow F and Melchinger AE (2012). Comparison of whole-genome prediction models for traits with contrasting genetic architecture in a diversity panel of maize inbred lines. BMC Genomics 13: 452.

Tang J, Yan J, Ma X, Teng W, et al. (2010). Dissection of the genetic basis of heterosis in an elite maize hybrid by QTL mapping in an immortalized $\mathrm{F}_{2}$ population. Theor. Appl. Genet. 120: 333-340.

Voorrips RE (2002). MapChart: software for the graphical presentation of linkage maps and QTLs. J. Hered. 93: 77-78.

Wang S, Basten CJ and Zeng ZB (2010). Windows QTL Cartographer 2.5. Department of Statistics, North Carolina State University, Raleigh, NC, USA. Available at http://statgen.ncsu.edu/qtlcart/WQTLCart.htm. Accessed March $10,2010$.

Xu S, Zhu D and Zhang Q (2014). Predicting hybrid performance in rice using genomic best linear unbiased prediction. Proc. Natl. Acad. Sci. USA 111: 12456-12461.

Zhang G, Wang X, Wang B, Tian Y, et al. (2013). Fine mapping a major QTL for kernel number per row under different phosphorus regimes in maize (Zea mays L.). Theor. Appl. Genet. 126: 1545-1553.

Zhang Z, Liu Z, Hu Y, Li W, et al. (2014). QTL analysis of kernel-related traits in maize using an immortalized F2 Population. PLoS One 9: e89645.

Zhou T, Gao C, Du L, Feng H, et al. (2014). Genetic analysis and QTL detection for resistance to white tip disease in rice. PLoS One 9: e106099. 